\title{
Health Professional
}

National Cancer Institute

\section{Source}

National Cancer Institute. Health Professional. NCI Thesaurus. Code C54067.

A person trained to work in any field of physical or mental health. 